\title{
Heavy Ion Physics in the RHIC Era
}

\author{
Peter M. Jacobs* \\ Lawrence Berkeley National Laboratory \\ Berkeley, California \\ U.S.A. 94720 \\ E-mail: 'pmjacobs@ibl. gov'
}

Abstract: This talk reviews the field of Relativistic Heavy Ion Physics and the search for phase transitions in strongly interacting matter. I will briefly discuss the established fixed target experimental programs, and then focus on what has been learned from the first run of the Relativistic Heavy Ion Collider at BNL.

\section{Introduction}

The phase diagram of QCD exhibits a rich structure (e.g. Fig. $\left.\underline{1}_{1}^{1}\right)$. At temperatures $T \gg \Lambda_{\mathrm{QCD}}$ the coupling between quarks and gluons is weak and a deconfined phase, known as the Quark-Gluon Plasma or $Q G P$, is expected to exist, whereas below $T \sim \Lambda_{\mathrm{QCD}}$ the coupling is strong and partons are confined within hadrons. Consequently, a confinementdeconfinement phase transition may occur at $T \sim 150-200 \mathrm{MeV}$. The compression of matter at low temperature but high baryon density also induces deconfinement, possibly into colour-superconducting or other exotic states [1]

A second phase transition, the restoration of chiral symmetry, likewise occurs at high energy density and may be coincident with the (de-)confinement transition. In the following I will often refer simply to the "phase transition", specifying when necessary which of the two is meant.

In the early universe, the confinement transition occured at high temperature and very low baryon density, at an age of about $10^{-5}$ seconds. A strongly first order transition may have had significant effects at this epoch [2i], perhaps generating primordial black holes, strange quark nuggets, or baryon asymmetries, the latter affecting primordial nucleosynthesis. The QGP phase may exist in the present universe in the cores of neutron stars, leading to unusual millisecond pulsar phenomena (e.g [3īi]).

The QCD phase diagram has also been studied on the lattice [i $\left[\begin{array}{l}1 \\ 1\end{array}\right]$. For three light, degenarate quarks the phase transition is first order, while for two flavours it is likely second order. For physical strange quark mass and $\mu_{\mathrm{B}}=0$ there may be a rapid cross over 


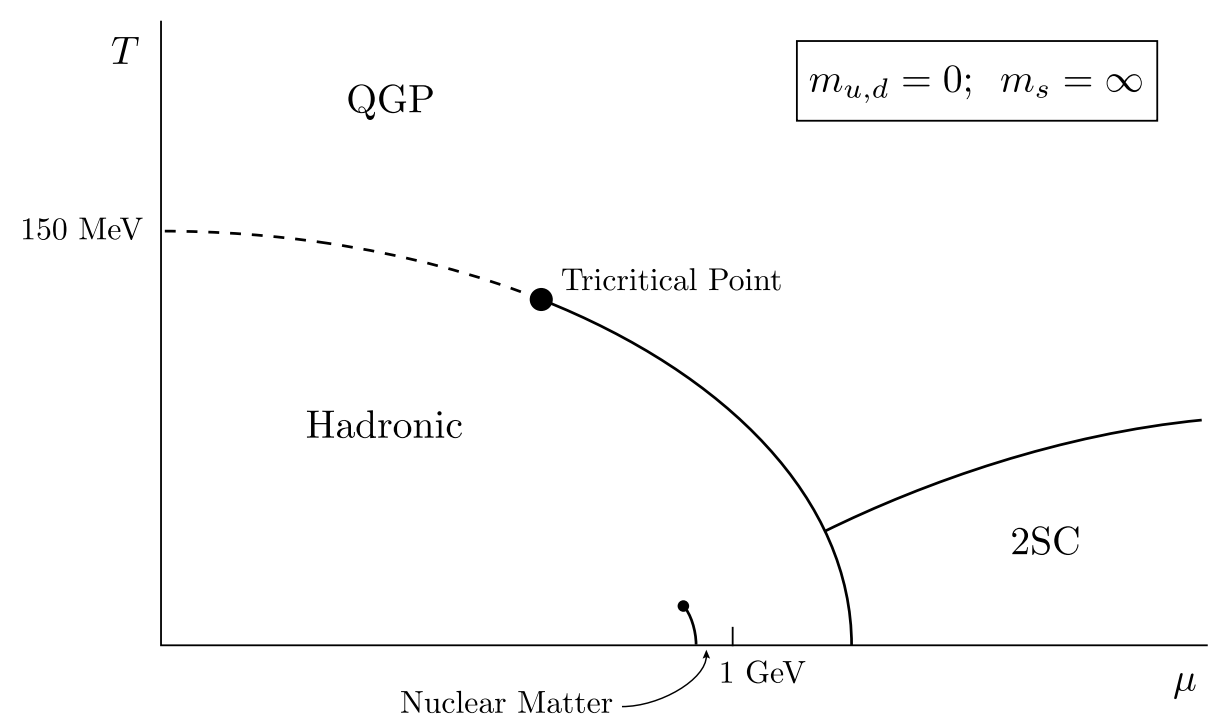

Figure 1: Example of the QCD phase diagram (temperature T vs. baryochemical potential $\mu_{\mathrm{B}}$ ) for two massless flavours [ilin]. Chiral symmetry is broken in the hadronic phase. "2SC" is a colorsuperconducting phase. The early universe descended from high $\mathrm{T}$ at extremely small $\mu_{\mathrm{B}}$. Neutron star cores have high $\mu_{\mathrm{B}}$ and very low $\mathrm{T}$.

rather than a phase transition, though this is not firmly established. Lattice calculations until recently were restricted to $\mu_{\mathrm{B}}=0$, but new techniques have been developed to probe

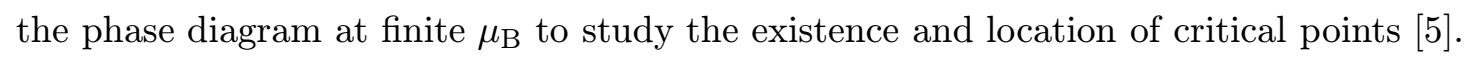

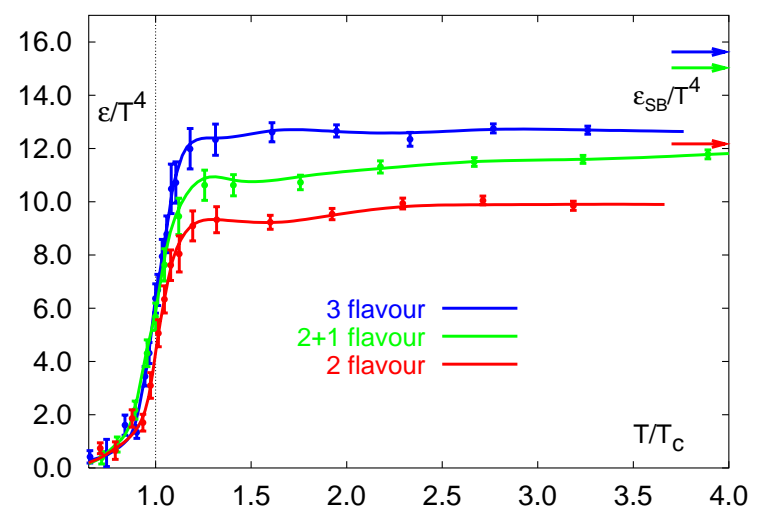

Figure 2: Lattice calculaton of energy density vs temperature for two- and three-flavour QCD [in]
Figure $\stackrel{\overline{2}}{2}$ shows a recent lattice calculation of the energy density $\epsilon$ as a function of temperature for two- and three-flavour QCD. There is a sharp transition at $\mathrm{T}_{\mathrm{C}}$ in all cases, indicating a transition in the number of underlying degrees of freedom. The Stefan-Boltzmann ideal gas limit $\epsilon_{\mathrm{SB}}$ has not yet been achieved at $\mathrm{T} / \mathrm{T}_{\mathrm{C}}=3.5$, i.e. residual interactions persist well above $\mathrm{T}_{\mathrm{C}}$. The critical energy density is $\epsilon_{\mathrm{C}}=$ $(6 \pm 2) \mathrm{T}_{\mathrm{C}}{ }^{4}$ and conversion to a physical scale gives $\mathrm{T}_{\mathrm{C}} \sim 175 \mathrm{MeV}$ and $\epsilon_{\mathrm{C}} \sim 1 \mathrm{GeV} / \mathrm{fm}^{3}$. This value of $\epsilon_{\mathrm{C}}$ is a useful benchmark for assessing the conditions achieved in laboratory experiments and I will return to it below.

One of the notable properties of the QCD phase transition is that it is the only phase transition predicted by the standard model that can potentially be studied in the laboratory [2i], through the collision of heavy nuclei at high energy. The study of relativistic heavy ion collisions at accelerators began thirty years ago at the Bevalac [6i considerably with the advent of fixed target heavy ion programs at the AGS at BNL and the SPS at CERN in the late '80s. Though positive identification of the Quark Gluon 
Plasma phase has proved elusive for the fixed target experiments, they have mapped out the experimentally accessible landscape in considerable detail and a number of suggestive results have emerged $[\bar{i}]$. A major step forward in the search for the Quark Gluon Plasma was taken with the commissioning of the Relativistic Heavy Ion Collider (RHIC) and its associated experiments at Brookhaven National Laboratory in summer 2000.

\section{What can be learned from heavy ion collisions?}

The collision of nuclei at high energy is a complex, highly dynamic process, ultimately generating hundreds (at the SPS) or thousands (at RHIC) of secondary particles. Following the initial impact of the incoming nuclei, a region of hot and dense matter is generated at mid-rapidity. In a central, head-on collision, only a small fraction of the incoming nucleons do not interact ("spectators") and continue down the beampipe. The interaction region immediately begins to expand longitudinally and transversely, cooling in the process, so that the highest energy density (and the best opportunity to generate a QGP) is achieved early in the reaction. With further expansion and cooling the matter hadronizes and forms a dense, interacting hadron gas. Eventually the temperature drops to a level that inelastic collisions between hadrons are rare, at which point the system has reached chemical freezeout and the relative population of various final state hadron species is established. After further expansion and cooling the system becomes so dilute that elastic scattering among hadrons is rare, at which point kinetic freezeout has been achieved and the final momentum spectra of hadrons observed in the detectors are established.

A natural question is whether the presence of a deconfined phase in the core of such a collision can be seen through the enormously complicated final state. There are a number of promising experimental observables to probe the state of the matter and the dynamics of the expansion (for a review see [i] i i ), among them:

- Observation of deconfinement: suppression of $\mathrm{J} / \psi$

- Measurement of energy density: jet energy loss

- Measurement of temperature: excess of virtual and real photons beyond hadronic sources

- Non-hadronic degrees of freedom: event-by-event fluctuations

- Rapid equilibration: large anisotropic flow in non-central collisions, strangeness enhancement

A system undergoing a phase transition exhibits threshold behaviour: the system passes sharply from one phase to another at particular values of the thermodynamic parameters. In heavy ion collisions the energy density, temperature or baryon density cannot be dialed in directly but can be varied indirectly through changes in $\sqrt{s_{\mathrm{NN}}}$, the centrality (impact parameter) of the collision, and the mass of the colliding nuclei. An important strategic component in the search for the QGP is the systematic study of the sensitive 

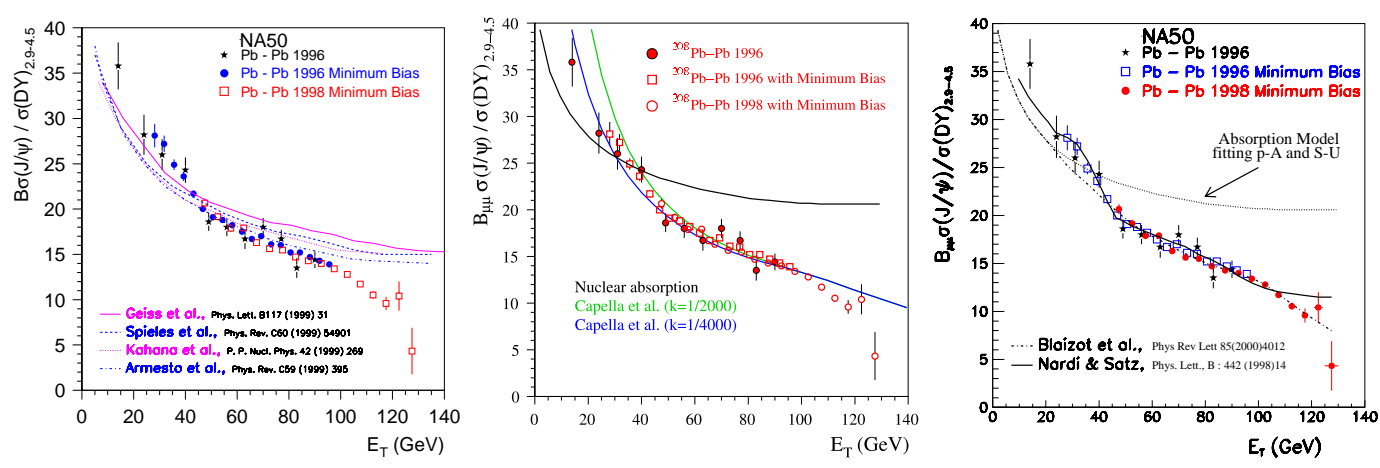

Figure 3: Ratio of $\mathrm{J} / \psi$ to Drell-Yan production cross sections vs $\mathrm{E}_{\mathrm{T}}$, from NA50 [1] $\overline{1}_{1}$. Same data appear in all panels, theory curves are discussed in text.

observables while varying the energy and centrality of the collisions and the size of the incoming nuclei.

Each of the observables under discussion thus far is nevertheless qualified to some extent: definitive discrimination of a deconfined plasma from a hot hadronic gas may not be achievable using any one observable. While the hope remains to find a "smoking gun" that unambiguously identifies deconfinement, the more likely scenario is that compelling evidence for the QGP will emerge as the most reasonable picture from the systematic study of a number of different observables, each sensitive to a different aspect of the collision.

\section{Fixed Target Experiments}

Beams of $\mathrm{Au}$ ions at $11.7 \mathrm{GeV} /$ nucleon (BNL AGS) and $\mathrm{Pb}$ ions at $158 \mathrm{GeV} /$ nucleon (CERN SPS) have been available for fixed target experiments since the early-mid '90s. A broad experimental program was carried out at both facilities and a rather complete picture

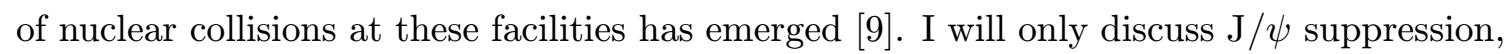
briefly summarizing the other main physics points from the SPS.

The suppression of the $\mathrm{J} / \psi$ yield as a signature of deconfinement in nuclear collisions was proposed by Matsui and Satz in 1986 [i1 100 : formation of cc mesons is suppressed due to colour Debye screening in the plasma. $\mathrm{J} / \psi$ suppression has been investigated experimentally in a series of studies by the NA38 and NA50 collaborations, using a di-muon spectrometer at the SPS [1]1],

Fig. ${ }^{3}-1$ shows the $\mathrm{J} / \psi$ production cross section in $\mathrm{Pb}+\mathrm{Pb}$ collisions as a function of the transverse energy $\left(\mathrm{E}_{\mathrm{T}}\right)$ in the collision $\left(\mathrm{E}_{\mathrm{T}}\right.$ measured in $\left.1.1<\eta_{\text {lab }}<2.3\right) . \quad \mathrm{E}_{\mathrm{T}}$ is related to the geometry of the nuclear collision: larger $\mathrm{E}_{\mathrm{T}}$ corresponds to smaller impact parameter or more "central" collisions. The $\mathrm{J} / \psi$ cross section is normalized to the measured Drell-Yan cross section at high invariant pair mass. The probability for any rare process (small cross section) scales in nuclear collisions as $\mathrm{N}_{\text {binary }}$, which applies at the SPS both

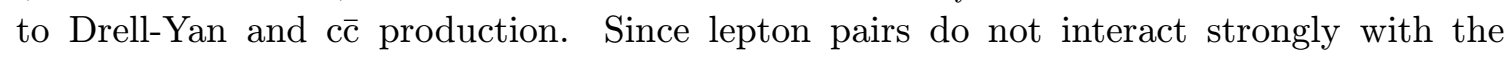
medium, the observed Drell-Yan cross section is also proportional to $\mathrm{N}_{\text {binary }}$ and is a suitable normalization to measure the suppression of the $\mathrm{J} / \psi$ cross section. By using the DrellYan and $\mathrm{J} / \psi$ cross sections measured in the same set of events, the variation of collision 
geometry (and corresponding variation of $\mathrm{N}_{\text {binary }}$ ) within the class of nuclear collisions falling into each $\mathrm{E}_{\mathrm{T}}$ bin is properly taken into account. I will return to this crucial issue of geometry and counting of binary collisions when discussing the production of high $p_{T}$ hadrons at RHIC.

Suppression of $\mathrm{J} / \psi$ production in nuclear collisions can arise from purely hadronic

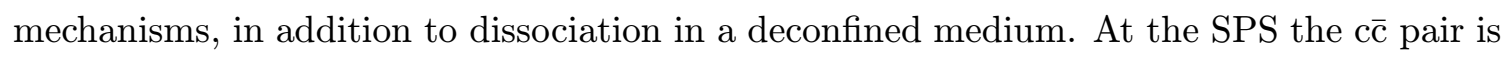
formed early in the collision, interacting with the trailing cold nuclear matter that continues to enter the collision zone (the suppression due to this mechanism is indicated on the right panel of Fig. ${ }_{3-1}^{3}$ as the "Absorption Model"). The formed $\mathrm{J} / \psi$ can also interact with produced hadrons in the final state. Both mechanisms generate the largest suppression at the highest $\mathrm{E}_{\mathrm{T}}$ (central collisions). The curves in the left and center panels of the figure show several calculations incorporating these purely hadronic effects. The data exhibit an additional "anomalous" suppression at the highest $\mathrm{E}_{\mathrm{T}}$ relative to these curves. Also apparent in the data are two "steps" at intermediate $\mathrm{E}_{\mathrm{T}}$, not reproduced by the hadronic calculations. The NA50 collaboration suggests that these steps are not hadronic in origin but are related to melting in the plasma of the more weakly bound $\psi \prime$ and $\chi_{\mathrm{c}}$ as the energy density passes successive thresholds [i] ${ }_{1}^{1} i_{1}^{1}$.

The right panel of Fig. 13 , compares the data to calculations in which the $\mathrm{J} / \psi$ is absorbed in regions of the collision where the energy density exceeds the threshold for deconfinement. This region covers a larger fraction of the reaction volume the more central the collision, producing greater suppression at higher $\mathrm{E}_{\mathrm{T}}$. The dissociation of $\psi \prime$ and $\chi_{\mathrm{c}}$ above lower thresholds ("steps") and energy density fluctuations associated with the high $\mathrm{E}_{\mathrm{T}}$ tail are also taken into account. In general, the steps and high $\mathrm{E}_{\mathrm{T}}$ behaviour are better described by the QGP models than the hadronic ones.

In the interest of space I will not discuss in detail other topics from fixed target experiments, but simply summarize the current understanding of $\mathrm{Pb}+\mathrm{Pb}$ collisions at the SPS (for details see [īit] and references therein):

- Hadron thermodynamics: system at mid-rapidity is baryon-rich $\left(\mu_{\mathrm{B}} \sim 250 \mathrm{MeV}\right)$, initial energy density $\epsilon \sim 3 \mathrm{GeV} / \mathrm{fm}^{3}$. There is evidence that equilibrium is achieved early in the collision.

- Enhancement of low mass di-electrons: evidence for chiral symmetry restoration?

- Direct photon yields beyond those from hadronic sources: radiation from plasma?

- Multistrange baryon enhancement, charmonium suppression: favour QGP over hadron gas scenario

The evidence for the observation of deconfinement at the SPS is suggestive but not definitive. Theoretical ambiguities persist for distinguishing the QGP from a hadron gas with the current data. If deconfinement has indeed been achieved at the highest SPS energy, it remains to be shown that the phase transition can be turned off at lower energy. It has been predicted that a tricritical point exists within the range of temperature and 
baryon density accessible to the SPS [i1, $\left[\begin{array}{l}1 \\ 1\end{array}\right]$ The SPS experimental program has therefore undertaken a study of nuclear collisions at lower $\sqrt{s_{\mathrm{NN}}}$, currently in progress.

\section{The Relativistic Heavy Ion Collider}

The Relativistic Heavy Ion Collider (RHIC) at Brookhaven National Laboratory is a new

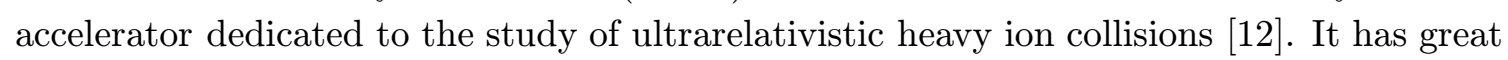
flexibility in beams and energies, colliding $\mathrm{Au}$ ions at energies up to $\sqrt{s_{\mathrm{NN}}}=200 \mathrm{GeV}$ and polarized protons up to $\sqrt{s}=450 \mathrm{GeV}$. $\mathrm{Pb}+\mathrm{Pb}$ collisions at the SPS were at $\sqrt{s_{\mathrm{NN}}}=17$ $\mathrm{GeV}$; RHIC therefore represents an order of magnitude increase in $\sqrt{s_{\mathrm{NN}}}$.

Relative to lower energies, the higher $\sqrt{s_{\mathrm{NN}}}$ at RHIC is expected to generate higher initial energy density and a longer-lived hot phase. New physics channels open up (jets, copious production of charm, B-quark production) and the statistics and kinematic reach of previously studied channels will be greatly extended, supplying important new tools for probing the system. The baryon density $\left(\mu_{\mathrm{B}}\right)$ at mid-rapidity should be much smaller than at lower energies, creating a system closer to the system studied on the lattice and the state of the early universe. The great flexibility in beams and energies at RHIC will allow a wide-ranging survey of reference data, an essential tool in the search for new phenomena.

The RHIC design for gold ion collisions specifies a top beam energy of $100 \mathrm{GeV} /$ nucleon $\left(\sqrt{s_{\mathrm{NN}}}=200 \mathrm{GeV}\right)$, with 57 bunches per ring and $10^{9}$ ions per bunch. Design store time is 10 hours and design luminosity $\mathcal{L}=2 \cdot 10^{26} \mathrm{~cm}^{-2} \mathrm{sec}^{-1}$. At first sight the design luminosity appears to be extremely small relative to other high energy colliders. However, for mass $\mathrm{A}$ and $\mathrm{B}$ of the colliding nuclei the hard scattering cross sections scale as A.B (=38809 for $\mathrm{Au}+\mathrm{Au}$ ), so that hard scattering rates in $\mathrm{Au}+\mathrm{Au}$ collisions at RHIC design luminosity are equivalent those of a $200 \mathrm{GeV}$ proton collider having $\mathcal{L}=8 \cdot 10^{30} \mathrm{~cm}^{-2} \mathrm{sec}^{-1}$.

The RHIC ring has six intersection regions, four of which are currently instrumented for physics: two small experiments, BRAHMS and PHOBOS, and two large experiments, PHENIX and STAR [1] $\left.{ }_{1}\right]$. The RHIC physics program began in the summer of 2000 with the collision of $\mathrm{Au}$ ions at $\sqrt{s_{\mathrm{NN}}}=130 \mathrm{GeV}$. Luminosity of $\mathcal{L}=2 \cdot 10^{25} \mathrm{~cm}^{-2} \mathrm{sec}^{-1}$ was achieved (10\% of design), with integrated luminosity for the run of a few $\mu \mathrm{b}^{-1}$. This modest integrated luminosity was nevertheless sufficient for a detailed first look into the physics of heavy ion collisions at RHIC. The second year of RHIC running, colliding Au ions at $\sqrt{s_{\mathrm{NN}}}=200 \mathrm{GeV}$, is underway.

After describing the characterization of event geometry at RHIC, I will discuss some of the major findings from the $130 \mathrm{GeV}$ run together with initial results from the $200 \mathrm{GeV}$ run. This is not a complete review, more details can be found in 991 .

\section{Geometry of Heavy Ion Collisions}

The size and shape of the interaction region can be changed by varying the impact parameter. The largest reaction volume and highest energy density are achieved for central (head-on) collisions. The impact parameter is of course not measurable, but observables 
correlated with the geometry of the collision can be used to bias the event selection in a controlled way.

All four RHIC experiments share a common device, the Zero Degree Calorimeter (ZDC) 迎需, for triggering, characterization of event geometry, and luminosity monitoring. The ZDCs are compact hadronic calorimeters located symmetrically at $\pm 18 \mathrm{~m}$. from the interaction region, downstream of the first accelerator dipole magnets that sweep all charge particles out of their acceptance. The ZDC therefore measures the number of spectator neutrons, which is correlated with event geometry for impact parameters less than about $6 \mathrm{fm}$ [i] $\left.\overline{1}_{\mathbf{1}}\right]$. For larger impact

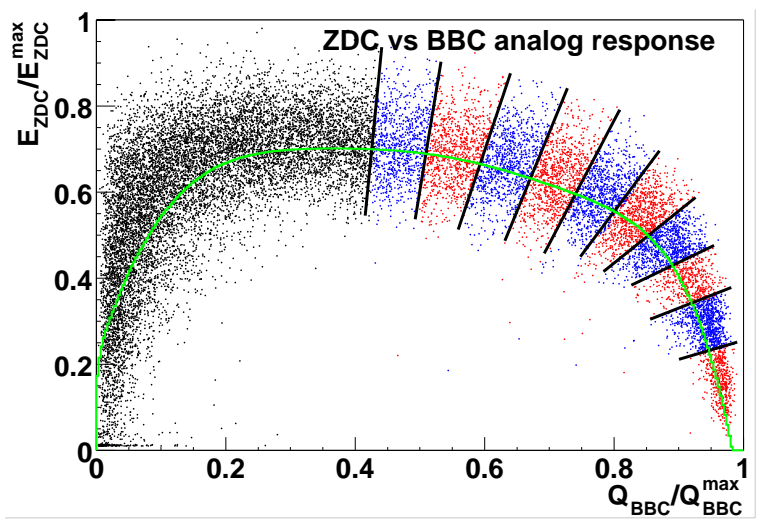

Figure 4: Event characterization from PHENIX: correlation of ZDC signal with forward multiplicity [1] parameters the event geometry is constrained using the correlation between ZDC signal and charged particle multiplicity in a forward region or at mid-rapidity.

Fig. 零 shows a typical correlation, in this case from PHENIX, of charged particle multiplicity $(3.0<|\eta|<3.9)$ with ZDC signal [i] $\left.{ }_{1}^{1} \overline{1} \mid\right]$. The most central collisions correspond to high multiplicity $\left(\mathrm{Q}_{\mathrm{BBC}} / \mathrm{Q}_{\mathrm{BBC}}{ }^{\max } \approx 1\right)$ and low ZDC energy $\left(\mathrm{E}_{\mathrm{ZDC}} / \mathrm{E}_{\mathrm{ZDC}}{ }^{\max } \ll 1\right)$, since very few spectator neutrons emerge from central collisions. The solid lines perpendicular to the ridge illustrate the division of the total cross section into event classes biased towards different centralities.

For a fixed collision geometry (fixed impact parameter), the number of incoming participants $\mathrm{N}_{\text {part }}$ (complement of the number of spectators) and the number of binary nucleonnucleon collisions $\mathrm{N}_{\text {binary }}$ can be calculated in a Glauber model that incorporates the nuclear

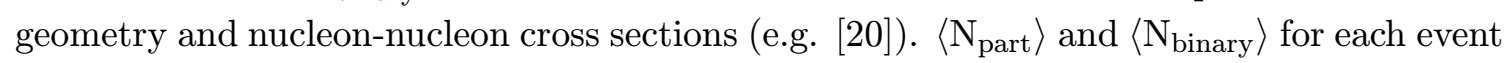
class in Fig. 集 are estimated by equating fractions of total cross section in the data and the geometrical model calculation. The systematic uncertainty of $\mathrm{N}_{\text {binary }}$ is $10-15 \%$ for the most central collisions. For events in the peripheral half of the multiplicity distribution the uncertainty is much larger and is difficult to assess, due to the uncertainties inherent in modelling multiplicity fluctuations.

\section{First Physics Results from RHIC}

\subsection{Baryon Density at Mid-rapidity}

The net baryon density at mid-rapidity is one of the essential parameters of collisions at RHIC, controlling where the phase diagram in Fig. ratio of yields at mid-rapidity of antiprotons to protons $(\overline{\mathrm{p}} / \mathrm{p})$ vs $\sqrt{s_{\mathrm{NN}}}$ for central collisions of heavy nuclei at the AGS, SPS and RHIC, and for $\mathrm{p}+\mathrm{p}$ collisions at various $\sqrt{s}$. The ratio increases dramatically with $\sqrt{s}$, reaching 0.6 at RHIC. STAR has also reported $\bar{\Lambda} / \Lambda \approx 0.7$ 


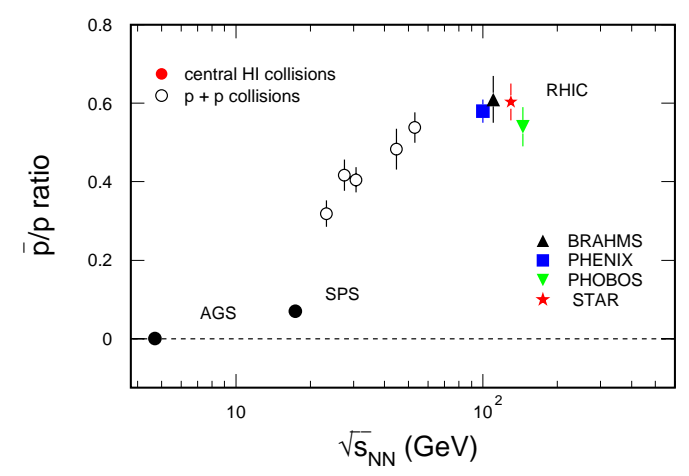

Figure 5: $\overline{\mathrm{p}} / \mathrm{p}$ ratio vs. $\sqrt{s_{\mathrm{NN}}}\left[\overline{1} \overline{7}_{1}\right]$. All RHIC data are at $\sqrt{s_{\mathrm{NN}}}=130 \mathrm{GeV}$ but are displaced horizontally for clarity.

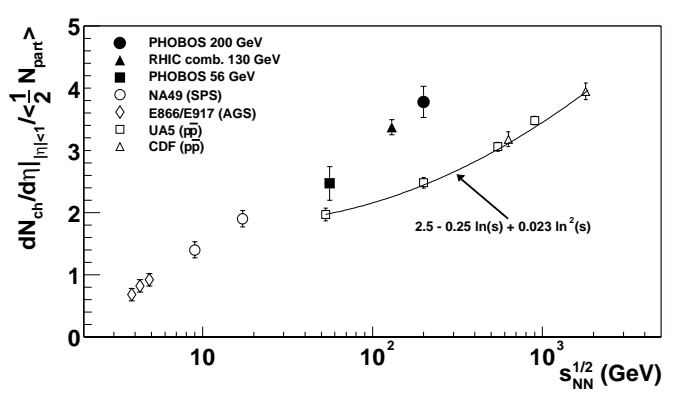

Figure 6: $d N_{c h} /\left.d \eta\right|_{\eta \sim 0}$ vs $\sqrt{s_{\mathrm{NN}}}$ for central nuclear collisions and $\overline{\mathrm{p}}+\mathrm{p}$ collisions [2] $\left.{ }^{[2} 2 \overline{-}\right]$.

and $\bar{\Xi} / \Xi \approx 0.8[\overline{1} \overline{1} \overline{8} \overline{8}]$. The system at RHIC is therefore almost baryon-free, approaching the vertical axis on Fig. $\underline{1}_{1}^{1}$. For precisely zero net baryon number the ratio is unity, not achieved at RHIC, thus a small baryon asymmetry persists at mid-rapidity. Future RHIC data will measure this asymmetry over wider phase space and address the transport mechanism that moves baryon number from the beam to mid-rapidity $(\delta y=5.5)$.

\subsection{Charged Particle Production}

The charged particle density near midrapidity, $d N_{c h} /\left.d \eta\right|_{\eta \sim 0}$, has been published by

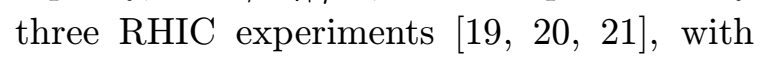
consistent values. For central $\mathrm{Au}+\mathrm{Au}$ at $\sqrt{s_{\mathrm{NN}}}$ $=130 \mathrm{GeV}$ the world average is $d N_{c h} /\left.d \eta\right|_{\eta \sim 0}=$

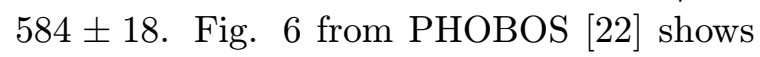
the dependence of $d N_{c h} /\left.d \eta\right|_{\eta \sim 0}$ (normalized to $\mathrm{N}_{\text {part }} / 2$ ) on $\sqrt{s_{\mathrm{NN}}}$ for heavy nuclei, including recent data at $200 \mathrm{GeV} \cdot \overline{\mathrm{p}}+\mathrm{p}$ collider data are also shown, exhibiting the wellknown $\ln (s)$ dependence. At $\sqrt{s}=130 \mathrm{GeV}$ the multiplicity per nucleon pair is $40 \%$ higher in $\mathrm{Au}+\mathrm{Au}$ collisions than in $\overline{\mathrm{p}}+\mathrm{p}$, demon-

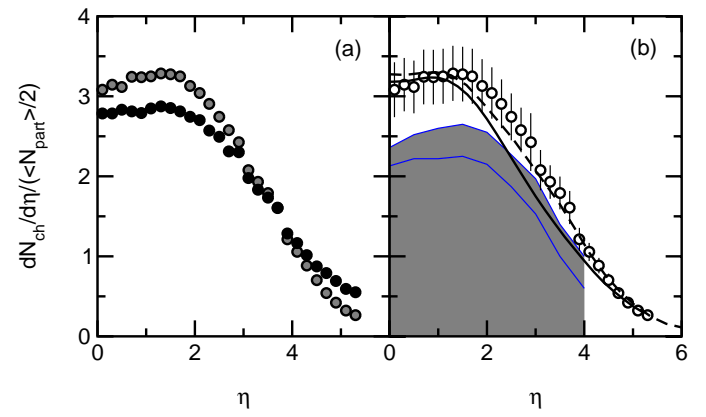

Figure 7: $d N_{c h} / d \eta$ at $\sqrt{s_{\mathrm{NN}}}=130 \mathrm{GeV}$ from PHOBOS [23i]. Left panel: central (open) and peripheral (filled) collisions. Right panel: comparison to models and $\overline{\mathrm{p}}+\mathrm{p}$ data (see text). strating that $\mathrm{Au}+\mathrm{Au}$ is not a simple superposition of more elementary collisions. The growth with $\sqrt{s}$ is also faster in nuclear collisions, which may be due to significant contribution of hard scattering to the multiplicity (hard collisions scale as $\mathrm{N}_{\text {binary }}$, whereas soft collisions scale approximately as $\mathrm{N}_{\text {part }}: \mathrm{N}_{\text {binary }} / \mathrm{N}_{\text {part }}=\frac{1}{2}$ for $\overline{\mathrm{p}}+\mathrm{p}, \mathrm{N}_{\text {binary }} / \mathrm{N}_{\text {part }} \approx \frac{1000}{350}$ for central $\mathrm{Au}+\mathrm{Au}$ ). The measured increase of $14 \%$ in $d N_{c h} /\left.d \eta\right|_{\eta \sim 0}$ from 130 to $200 \mathrm{GeV}$

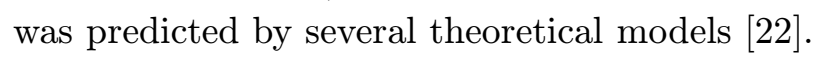

The density of charged particles $d N_{c h} / d \eta$ over the full phase space of RHIC is shown in Fig. $\overline{7}_{1}$ from PHOBOS [i2 $\left.2 \overline{3}\right]$, again normalized to $\mathrm{N}_{\text {part }}$. In the full phase space there are $4200 \pm 470$ charged particles produced in the most central collisions. The left panel shows $d N_{c h} / d \eta$ for central (0-6\% of geometric cross section, open symbols) and mid-peripheral 
collisions (35-45\%, filled symbols). A plateau extends from $\eta=0$ to $\eta \sim 2$, falling off rapidly towards beam rapidity. Central collisions exhibit an excess multiplicity per participant pair of $10-15 \%$ relative to mid-peripheral, the difference primarily in the plateau around midrapidity. The right panel compares the central data to the HIJING [24in] (solid curve) and AMPT [20 $2 \bar{j}$ ] (dashed curve) models. AMPT, consisting of a parton cascade, string fragmentation, and hadronic rescattering in a transport model, describes the full pseudorapidity distribution well. The right panel also shows a distribution interpolated to 130 $\mathrm{GeV}$ from $\mathrm{p}+\mathrm{p}$ and $\overline{\mathrm{p}}+\mathrm{p}$ data (filled band). The enhancement of $d N_{c h} /\left.d \eta\right|_{\eta \sim 0}$ in nuclear collisions relative to $\bar{p}+p$ is evident, with the excess again situated principally in the plateau.

\subsection{Bjorken Energy Density}

Within the framework of relativistic hydrodynamics, Bjorken proposed that for a boostinvariant source the maximum energy density achieved at mid-rapidity in central nuclear collisions could be estimated from the observed final $\mathrm{E}_{\mathrm{T}}$ or charged particle density [i2 $\left.\overline{2}_{\mathrm{G}}\right]$ :

$$
\epsilon_{\mathrm{Bj}}=\frac{1}{\pi R^{2} \tau} \frac{d \mathrm{E}_{\mathrm{T}}}{d y}
$$

where $R \propto A^{\frac{1}{3}}$ is the nuclear radius and $\tau$ is a formation time, usually taken as $1 \mathrm{fm} / \mathrm{c}$. The applicability of the boost-invariant approximation can be gauged from the region near $\eta=0$ in Fig. $\bar{\tau}_{-}$. For central $\mathrm{Au}+\mathrm{Au}$ at RHIC, from an $\mathrm{E}_{\mathrm{T}}$ measurement PHENIX obtained $\epsilon_{\mathrm{Bj}}=4.6 \mathrm{GeV} / \mathrm{fm}^{3}[2 \overline{2} \overline{7}]$, whereas STAR used charged particle distributions to calculate $\epsilon_{\mathrm{Bj}}=4.5 \mathrm{GeV} / \mathrm{fm}^{3}$. In comparison, an $\mathrm{E}_{\mathrm{T}}$ measurement for central $\mathrm{Pb}+\mathrm{Pb}$ at the SPS yielded $\epsilon_{\mathrm{Bj}}=3.0 \mathrm{GeV} / \mathrm{fm}^{3}[2 \overline{2} \overline{8}]$. The energy density at the phase transition on the lattice is $\epsilon_{\mathrm{C}} \sim 1 \mathrm{GeV} / \mathrm{fm}^{3}$ (see Section iiin). The values of $\epsilon_{\mathrm{Bj}}$ obtained for central collisions at RHIC and the SPS thus suggest that the energy density needed for the phase transition has been achieved. However, an important assumption in this analysis is that the hydrodynamic treatment is valid, i.e. that local equilibrium has been achieved at a time $\tau=1$ $\mathrm{fm} / \mathrm{c}$. I now turn to observables that address whether and when equilibrium is established in the collision.

\subsection{Kinetic Freezeout}

Kinetic freezeout occurs when elastic scattering among secondary hadrons effectively stops, which occurs very late in the collision. At this point the final transverse momentum spectra are established. Figure sis shows transverse momentum spectra of identified pions, kaons

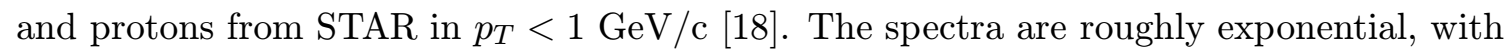
a "slope parameter" that depends on the particle mass: the more massive the particle, the stiffer the spectrum. This behaviour is naturally explained in a hydrodynamic picture, in which the final state hadrons are radiated from a fluid element at finite temperature which has finite transverse velocity ("radial flow"): at modest (non-relativistic) momenta, the common velocity boost corresponds to a stiffer momentum spectrum for the more massive 


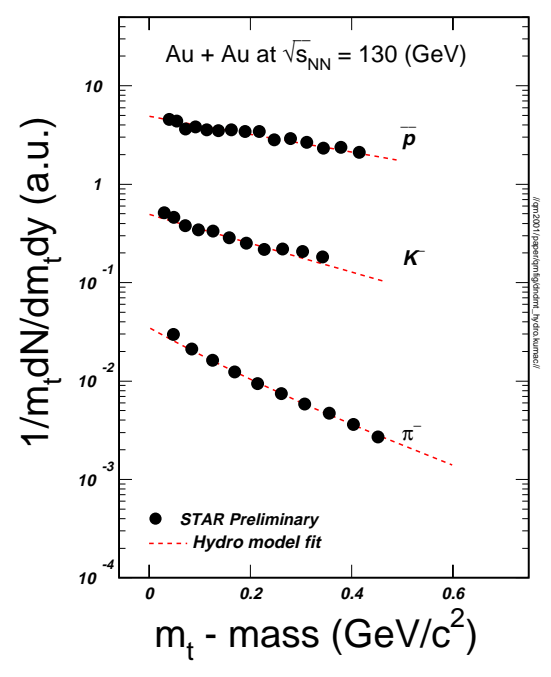

Figure 8: Invariant $p_{T}$ spectrum of $\pi^{-}, \mathrm{K}^{-}$

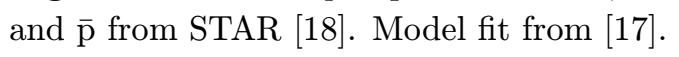

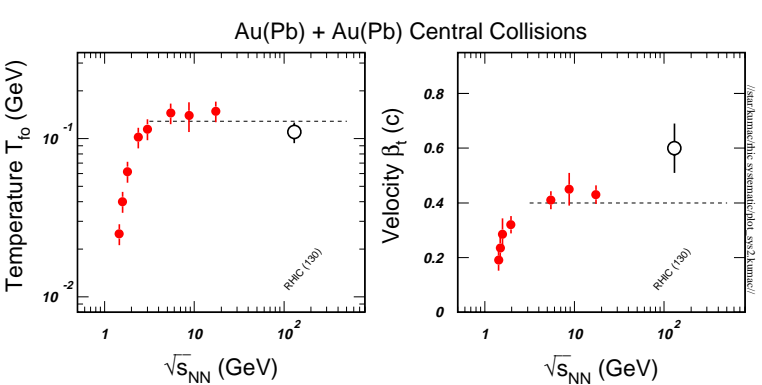

Figure 9: Temperature and transverse flow velocity vs $\sqrt{s_{\mathrm{NN}}}$ for central collisions of heavy nuclei $1 \overline{1}$ in.

particle. Also shown in the figure is a fit to the three spectra from a hydrodynamic model incorporating radial flow [i $[\bar{i}]$.

Figure $\bar{g}_{-1}$ is a systematic compilation of kinetic freezeout temperature $T_{\text {fo }}$ and transverse velocity $\beta_{\mathrm{t}}$ from similar hydrodynamic fits to hadronic spectra in nuclear collisions at energies from the Bevalac to RHIC [i] $\left.\bar{z}_{1}\right]$. T $\mathrm{T}_{\mathrm{fo}}$ and $\beta_{\mathrm{t}}$ saturate at $\sqrt{s_{\mathrm{NN}}} \sim 5 \mathrm{GeV}$, but $\beta_{\mathrm{t}}$ appears again to grow between the SPS and RHIC. Transverse flow is therefore larger at RHIC than at lower energies, in other words the expansion at kinetic freezeout is more explosive, indicating a high pressure earlier in the collision.

\subsection{Chemical Freezeout}

Chemical freezeout occurs when inelastic collisions effectively stop, at which point the relative yields of the different hadron species are established. Chemical freezeout occurs at a higher temperature, and therefore an earlier time, than kinetic freezeout. Several groups have studied the ratio of yields of various hadron species in a simple thermodynamic framework, incorporating a partition function of the spectrum of hadrons and a limited number of thermodynamic parameters (temperature; strangeness, baryon, and isospin chemical potentials). These simple models have been very successful at describing hadronic yields in a variety of colliding systems [20 $\underline{2} \overline{9}]$.

Fig. 'I] ${ }_{1}^{1}$ ' shows one example, in which various yield ratios measured by STAR are compared to the values obtained in a thermal model fit [i] $\left.\bar{i}_{-1}\right]$. The thermal model fits work alarmingly well given the simplicity of their assumptions. Typical fits to RHIC data yield chemical freezeout temperature $\mathrm{T}_{\text {chem }} \sim 175-200 \mathrm{MeV}, \mu_{\mathrm{B}} \sim 50 \mathrm{MeV}$ and $\mu_{\mathrm{S}} \sim 0$. These values are near the phase boundary on the vertical axis of Fig. 11. A purely hadronic mechanism for achieving equilibrium with these parameters seems unlikely: the equilibration time for strangeness in a hadron gas is about $50 \mathrm{fm} / \mathrm{c}\left[\mathbf{3} \underline{30}_{0}^{\prime}\right]$. Thus, the thermal 


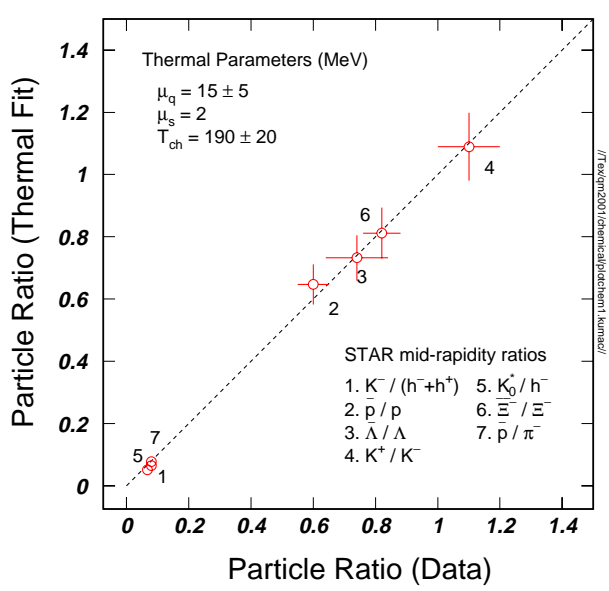

Figure 10: Yield ratios for various hadron species from STAR. Horizontal axis is data, vertical axis is thermal model fit [i]

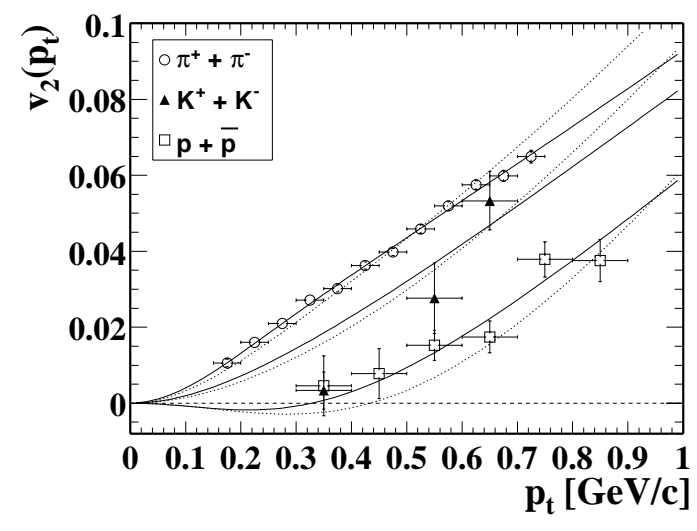

Figure 11: $v_{2}$ vs. $p_{T}$ for pions (open circles), kaons (triangles) and $\overline{\mathrm{p}}+\mathrm{p}$ (open squares) from STAR. Curves are fits using hydrodynamics-inspired models [34]"

model fits suggest that equilibrium is achieved by the time of chemical freezeout, and

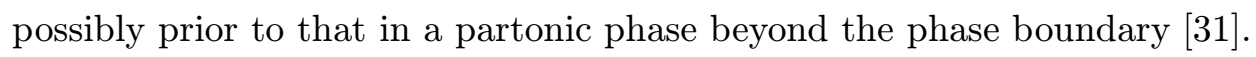

\subsection{Anisotropic Flow}

Non-central nuclear collisions generate an asymmetric reaction zone. If the constituents of the system rescatter during expansion, the initial spatial asymmetry will be transformed into an asymmetry in momentum space due to the azimuthally varying initial pressure gradient. The asymmetry is generated very early and is diluted as the system expands, meaning that a momentum space asymmetry correlated with the orientation of the reaction

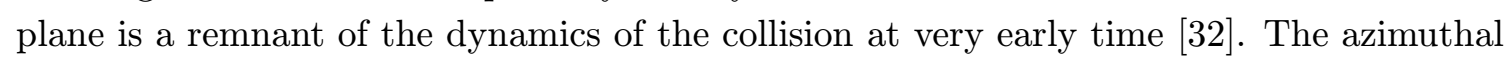
orientation of the reaction plane itself can be determined with good precision in these high multiplicity events [3i3i]. Anisotropic flow is quantified using a Fourier analysis of the azimuthal distribution in momentum space. The focus of flow studies so far at RHIC has been on the second order elliptic flow coefficient $v_{2}=\langle\cos (2 \phi)\rangle$, where $\phi=\tan ^{-1}\left(\frac{p_{y}}{p_{x}}\right)$.

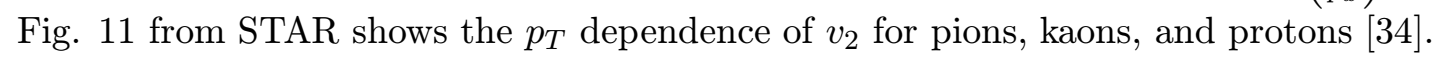
Again a dependence of the $p_{T}$ distribution on particle mass is seen and the same hydrodynamic argument pertains as with radial flow: in locally equilibrated hydrodynamics, hadrons are radiated from fluid elements which themselves have a velocity boost, generating a mass-dependent $p_{T}$ distribution for the radiated hadrons. Also shown in the figure are two hydrodynamics-inspired model fits, one of which (solid line) fits the mass and $p_{T}$

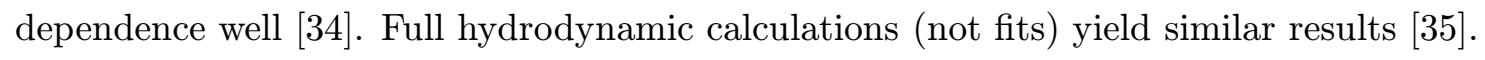

The excellent agreement between hydrodynamic calculations and the measured $v_{2}$, which probes the system at early time, indicates that local equilibrium is achieved very early in the collision, at high energy density. Work is underway to quantify the constraints that such measurements and model studies can put on the underlying Equation of State and the time at which equilibrium is achieved. 


\subsection{High $\mathrm{p}_{\mathrm{T}}$}

Due to the larger $\sqrt{s_{\mathrm{NN}}}$, the rate of jet production in nuclear collisions at RHIC is much greater than at the SPS. Following an initial unpublished suggestion by Bjorken, various

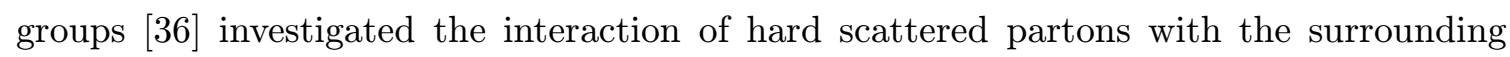
medium in nuclear collisions. They found that a parton traversing dense matter will lose energy ("jet quenching"), principally via gluon brehmsstrahlung, and that the energy loss will grow as $\mathrm{dE} / \mathrm{d} x \sim x^{2}$ due to coherence effects. The magnitude of $\mathrm{dE} / \mathrm{d} x$ in a deconfined medium could be of order $1 \mathrm{GeV} / \mathrm{fm}$, considerably larger than in hadronic matter [3isin]. Thus, the jet quenching phenomenon may be used to probe the energy density of the surrounding medium and is a potential signature for deconfinement.

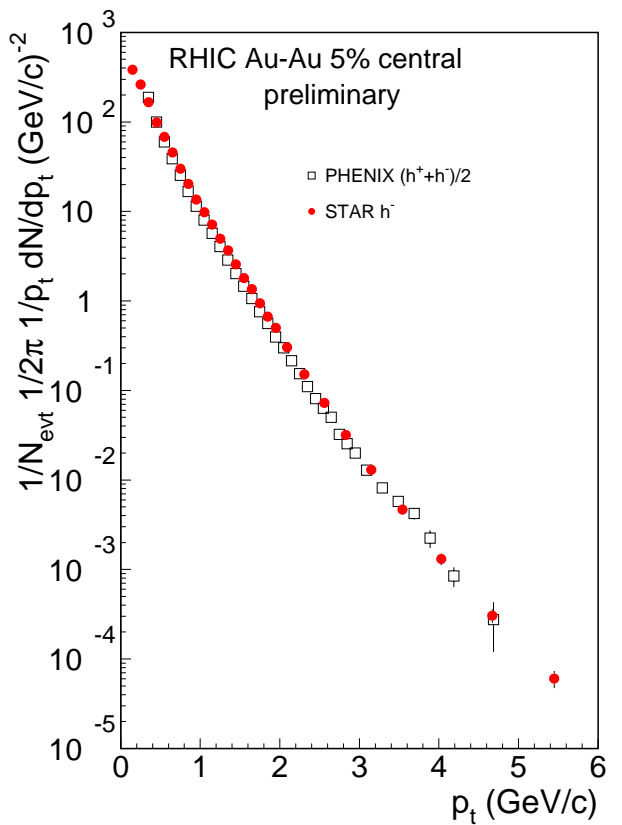

Figure 12: Invariant $p_{T}$ spectrum for charged hadrons from STAR "i $\overline{1}, \bar{\eta}$ and PHENIX [42]' Figure from [4i

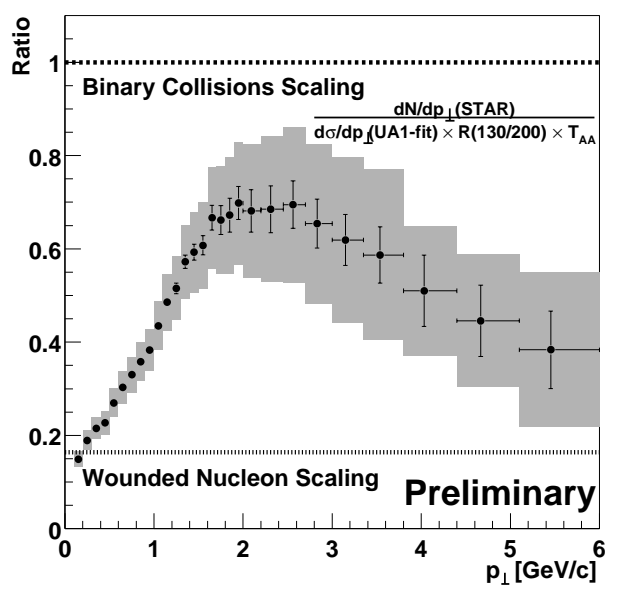

Figure 13: $R_{A A}\left(p_{T}\right)$ for central collisions vs $p_{T}$ for negative hadrons from STAR [1] $\left.1 \overline{1}^{\prime}\right]$, reference data from UA1.

Reconstruction of jets with good energy resolution in the high multiplicity environment at RHIC is extremely difficult, due to the complexity of the underlying event. However, the full jet is not the appropriate observable in any case: the radiated gluons will fall within the jet cone and the total jet energy will be insensitive to $\mathrm{dE} / \mathrm{d} x$. Rather, jet quenching corresponds to a softening of the jet fragmentation, which can be observed as a suppression of the yield of leading hadrons for finite $\mathrm{dE} / \mathrm{d} x\left[3 \overline{7}^{2}\right]$.

Suppression of $\mathrm{J} / \psi$ at the SPS was quantified by normalizing to the Drell-Yan cross section measured in the same collisions (Section isi.j). There is no similar process available for jet quenching at RHIC and the measurement of hadron suppression at high $p_{T}$ requires data from a reference system in which jet quenching is not expected to occur. The most desirable reference would be data from nucleon-nucleon collisions at $\sqrt{s}=130 \mathrm{GeV}$. These do not exist, unfortunately, and interpolation to $130 \mathrm{GeV}$ using $p_{T}$ spectra measured at 
the ISR and $\mathrm{Sp} \overline{\mathrm{p}} \mathrm{S}$ is necessary.

In the absence of any nuclear effects, the rate of hard processes is proportional to $\mathrm{N}_{\text {binary }}$. Hadron suppression can therefore be quantified by the deviation from unity of the

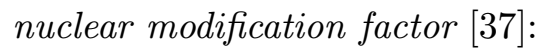

$$
R_{A A}\left(p_{T}\right)=\frac{d \sigma_{\mathrm{AA}} / d y d^{2} p_{T}}{\left\langle\mathrm{~N}_{\text {binary }}\right\rangle d \sigma_{\mathrm{pp}} / d y d^{2} p_{T}}
$$

There are nuclear effects other than jet quenching that can modify $R_{A A}\left(p_{T}\right)$ at $p_{T} \sim$ few

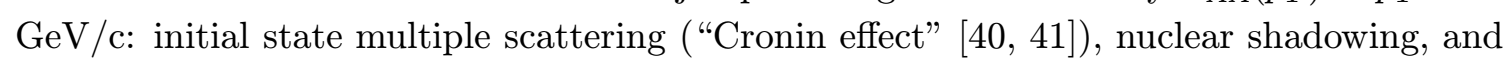
radial flow. A systematic assessment of these additional effects, most importantly via proton-nucleus collisions, is necessary to isolate definitively the effects of energy loss in dense matter.
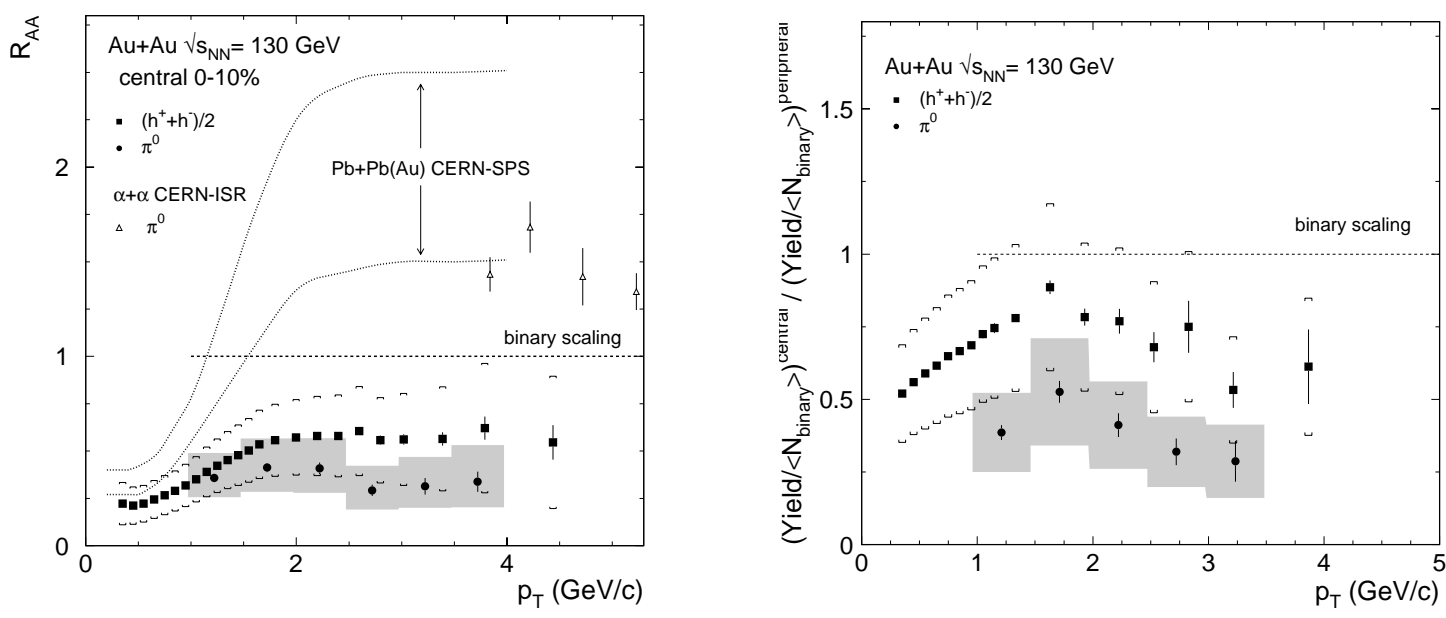

Figure 14: $R_{A A}\left(p_{T}\right)$ for central collisions vs $p_{T}$ for charged hadrons and $\pi^{0}$ from PHENIX [42 Left panel: normalized to nucleon-nucleon collisions. Right panel: normalized to peripheral nuclear collisions.

Fig. 112is shows the invariant $p_{T}$ distribution of charged hadrons for central $\mathrm{Au}+\mathrm{Au}$

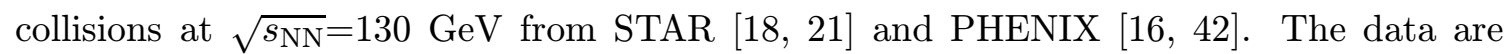
in agreement between the two experiments 7 to within $20 \%$. Fig. 13 , from STAR shows $R_{A A}\left(p_{T}\right)$ with respect to UA1 data [ī $\left.\overline{3} \bar{g}\right]$ extrapolated to $130 \mathrm{GeV}$. The error bars indicate the systematic uncertainties on the STAR measurement while the grey boxes show the total systematic uncertainty, including the extrapolation of UA1 data and the estimation of $\left\langle\mathrm{N}_{\text {binary }}\right\rangle$. Two simple limits are indicated in the figure: "Wounded Nucleon Scaling" (scaling with $\mathrm{N}_{\text {part }}$ ) and "Binary Collisions Scaling" (scaling with $\mathrm{N}_{\text {binary }}$, corresponding to $\left.R_{A A}\left(p_{T}\right)=1\right)$. Wounded nucleon scaling is valid at very low $p_{T}$ where the cross section is largest; this corresponds to the approximate scaling of the event multiplicity with $\mathrm{N}_{\text {part }}$ as seen in Fig. 'i: left panel. At high $p_{T}$, Binary Collisions scaling is not achieved, and at $p_{T} \sim 5.5 \mathrm{GeV}$ a suppression factor of about $50 \%$ is observed. 
Fig. $\overline{1} \overline{1} \overline{4}_{\mathrm{v}}^{\prime}$ left panel, shows $R_{A A}\left(p_{T}\right)$ from PHENIX for both charged hadrons and $\pi^{0}[i \overline{2} \overline{2} \overline{2}]$, where the reference now incorporates both ISR and UA1 data. Similar features at high $p_{T}$ are observed as in Fig. 'i] $\overline{1}_{3}^{\prime}$, though $R_{A A}\left(p_{T}\right)$ for charged hadrons from STAR decreases at high $p_{T}$ whereas that from PHENIX saturates. The suppression of the $\pi^{0}$ yield is even larger than that of charged hadrons. Also shown are $R_{A A}\left(p_{T}\right)$ measured in $\mathrm{Pb}+\mathrm{Pb}$ collisions at the SPS and $\alpha+\alpha$ collisions at the ISR. Both rise above unity at high $p_{T}$, in contrast to the RHIC data, indicating that the dominant effect in these cases is initial state multiple scattering. The right panel of the figure shows $R_{A A}\left(p_{T}\right)$ calculated purely from $\mathrm{Au}+\mathrm{Au}$ measurements at RHIC, in which the reference system (denominator of $R_{A A}\left(p_{T}\right)$ ) is very peripheral nuclear collisions. The same features are again seen: initial rise with increasing $p_{T}$ fol-

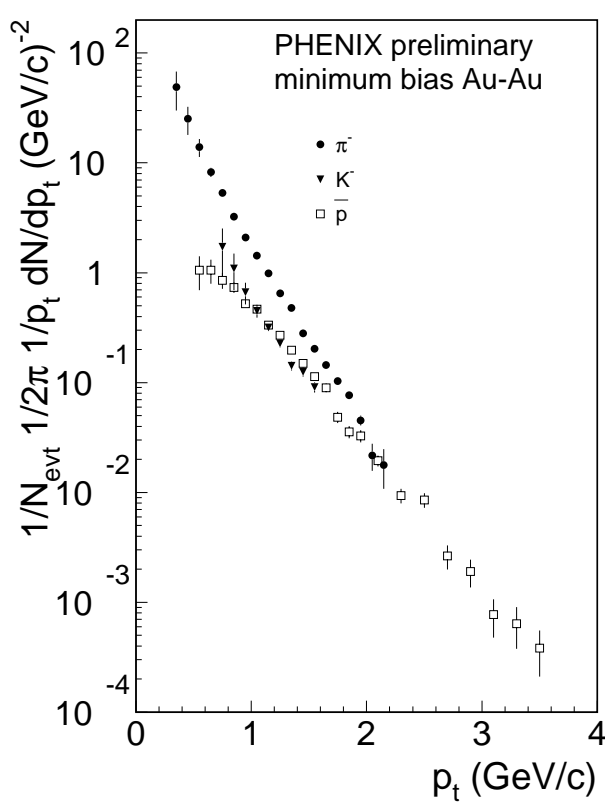

Figure 15: Invariant $p_{T}$ of $\pi^{-}, \mathrm{K}^{-}$and

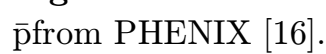
lowed by saturation below unity at the highest $p_{T}$ measured.

The difference in suppression factors between charged hadrons and $\pi^{0}$ measured by PHENIX is elucidated by Fig. $1 \overline{1} \overline{1}_{v}^{1}$, which shows invariant $p_{T}$ spectra for identified charged pions, kaons and $\overline{\mathrm{p}}$ at high $p_{T}$ for minimum bias $\mathrm{Au}+\mathrm{Au}$ collisions. At $2 \mathrm{GeV}$ the $\overline{\mathrm{p}}$ yield equals that of pions and apparently will exceed it at higher $p_{T}$. This is an unexpected result in light of jet fragmentation data, in which the proton yield never exceeds $20 \%$ of the charged hadron yield. Though $p_{T}=2 \mathrm{GeV} / \mathrm{c}$ is hardly in the realm of perturbative processes, if this effect persists at higher $p_{T}$ it will be the sign of something quite new and unexpected, perhaps exotic jet fragmentation in the nuclear medium, new mechanisms of baryon production at high $p_{T}$ [43i] , or extremely explosive radial flow. Both PHENIX and STAR will be able to address this question at much higher $p_{T}$ in the second year of RHIC running.

Finally, I return the the question of elliptic flow, this time at high $p_{T}$. In non-central collisions the interaction zone is asymmetric. For a hard partonic scattering in such an event, the length of matter $L$ through which the parton travels to escape the medium will depend on the azimuthal orientation of its momentum with respect to the reaction plane. If the parton interacts significantly with the matter, its energy loss and the resulting suppression of the leading hadrons from its fragmentation will depend on $L$, i.e. a finite azimuthal correlation will persist at high $p_{T}$ between leading hadrons and the reaction

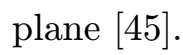

Fig. '1] $1{ }_{1}^{\prime}$ from STAR shows $v_{2}$ as a function of $p_{T}$ for charged particles from minimum bias $\mathrm{Au}+\mathrm{Au}$ collisions. In the left panel the data are compared to a hydrodynamic calcula-

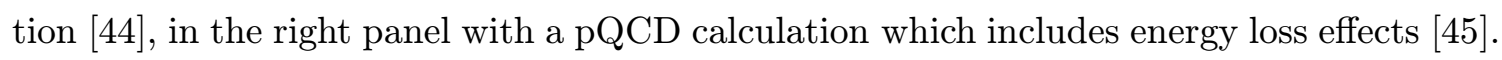
As in Fig. '1] $11_{1}^{\prime}$, at $p_{T}<2 \mathrm{GeV} / \mathrm{c}$ the hydrodynamic calculation agrees extremely well with 

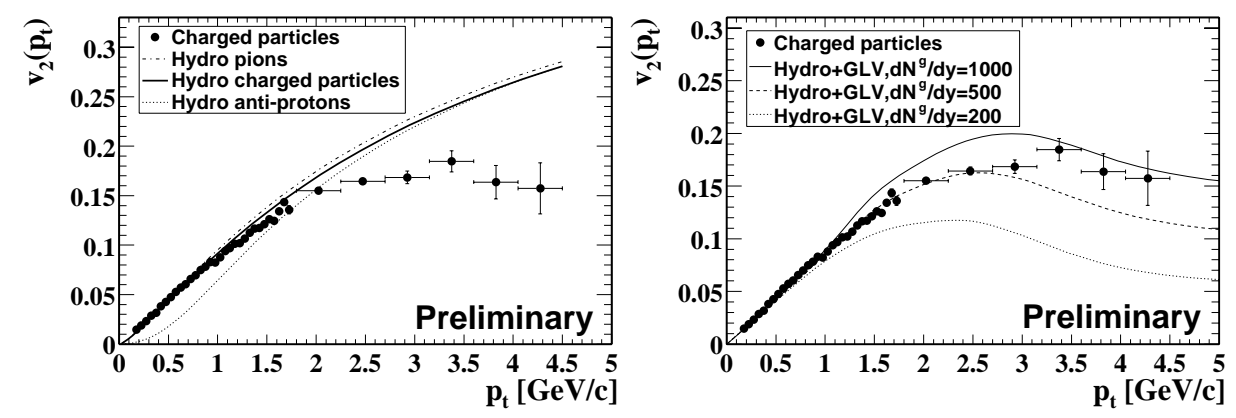

Figure 16: $v_{2}$ for charged hadrons from minimum bias collisions from STAR [1] described in text.

the data, but the data saturate and diverge from the calculation at higher $p_{T}$. Finite $v_{2}$ persists up to $p_{T}=4.5 \mathrm{GeV} / \mathrm{c}$ and can be described by the pQCD calculation incorporating energy loss and a high initial gluon density. However, the agreement at low $p_{T}$ with the hydrodynamic limit and deviation from it at high $p_{T}$ could be due simply to the changing contribution of soft and hard processes to the spectrum. Measurement of $v_{2}$ at yet higher $p_{T}$ will help to disentangle these issues.

The inclusive spectrum and $v_{2}$ at high $p_{T}$ are two rather different measurements, both potentially sensitive to the jet quenching phenomenon. As the RHIC experiments map out the high $p_{T}$ sector, pushing farther into the perturbative regime, both observables must be understood within a common theoretical framework. This promises to be a powerful approach to disentangle jet quenching from other nuclear effects, allowing it to be used as a diagnostic tool of the state of matter generated in the collision.

\subsection{Summary of physics results from the first year of RHIC}

The first year of physics running at RHIC was an unqualified success. The collider and all four experiments worked well and study of the physics of nuclear collisions at RHIC energies is well underway.

Studies at low $p_{T}$ show that the baryon density at mid-rapidity for nuclear collisions is very low but finite. From transverse momentum spectra, hadron yields, and elliptic flow, there is strong evidence that equilibrium is achieved early in the collision, at high energy density. The initial energy density is estimated to be $\sim 4.5 \mathrm{GeV} / \mathrm{fm}^{3}$. Radial expansion late in the collision is explosive, indicating high early pressure.

Studies up to $p_{T} \sim 5-6 \mathrm{GeV} / \mathrm{c}$ show evidence for the suppression of hadron yields in central collisions, perhaps the first hint of the jet quenching phenomenon in dense matter. The existence of finite elliptic flow at high $p_{T}$ may also be related to jet quenching, and studying both phenomena at yet higher $p_{T}$ is an urgent task. The yield of anti-protons equals that of pions at $p_{T}=2 \mathrm{GeV}$, and if this continues to higher $p_{T}$ it may indicate interesting new phenomena in jet fragmentation or high $p_{T}$ baryon production.

\section{Outlook}

The second year of RHIC running began in the summer of 2001. Collisions at full energy 
$\left(\sqrt{s_{\mathrm{NN}}}=200 \mathrm{GeV}\right)$ were achieved, and at the time of writing design luminosity is on the horizon. STAR and PHENIX have added several major new subsystems. Prospects for year 2 physics from RHIC include much higher $p_{T}$ spectra and correlations, the search for thermal radiation and the first look into $\mathrm{J} / \psi$ physics from PHENIX, a detailed look at multistrange baryon production from STAR, and the first run with polarized protons, the latter also generating key reference data for interpreting the heavy ion results. Also on the menu in the coming year are proton- (or deuteron)-nucleus collisions and nuclear collisions at different energies and masses to map out the systematics. New physics is emerging at RHIC and the future prospects are exciting.

\section{References}

[1] K. Rajagopal and F. Wilczek, hep-ph/0011333!

[2] D. Boyanovsky, hrep-ph/0102120!

[3] N. K. Glendenning and F. Weber, astro-ph/0003426

[4] F. Karsch, hep-ph/0103314;

[5] Z. Fodor and S. D. Katz, hep-1at/0104001, hep-lat/0106002:

[6] J. Gosset et al., iPhys. Rev. $\mathbf{\mathbf { 1 }} \mathbf{6}\left(\overline{1977)} 62_{1} ;\right.$ H.H. Gutbrod, A.M. Poskanzer, and H.G. Ritter, 'RepProg Phys $\mathbf{5} 2(1989) 1267$

[7] U. Heinz and M. Jacob, nucl-th/0002042i, CERN Press release 2000.02.10.

[8] J. W. Harris and B. Muller, Ann. Rev. Nucl. Part. Sci. $\mathbf{4} \mathbf{6}(\overline{1} \overline{9} 96)$

[9] Proceedings of Quark Matter 2001, Stony Brook, N.Y., Jan. 15-21, 2001. Nucl. Phys. A, in press..

[10] T. Matsui and H. Satz, iPhys. Lett.

[11] M. C. Abreu et al. (NA50 Collaboration), 'Phys. Lett. B $\mathbf{4} 50$ 0 1999$) 456$

[12] T. Roser, Proceedings of Quark Matter 2001, Stony Brook, N.Y., Jan. 15-21, 2001. Nucl. Phys. A, in press..

[13] BRAHMS: www4.rcf.bnl.gov/brahms/WWW/brahms.html; PHOBOS: phobos-srv.chm.bnl.gov; PHENIX: www.phenix.bnl.gov; STAR: www.star.bnl.gov.

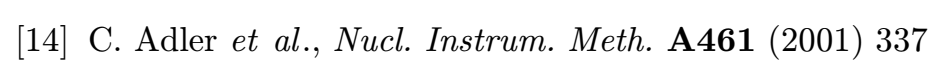

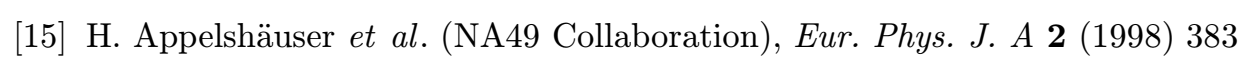

[16] W. A. Zajc et al. (PHENIX Collaboration), Proceedings of Quark Matter 2001, Stony Brook, N.Y., Jan. 15-21, 2001. Nucl. Phys. A, in press.; nucicex/0106001.

[17] N. Xu and M. Kaneta, Proceedings of Quark Matter 2001, Stony Brook, N.Y., Jan. 15-21, 2001. Nucl. Phys. A, in press.; nucici-ex/0104021;

[18] J.W. Harris et al. (STAR Collaboration), Proceedings of Quark Matter 2001, Stony Brook, N.Y., Jan. 15-21, 2001. Nucl. Phys. A, in press..

[19] B. B. Back et al. (PHOBOS Collaboration), 'Phys. Rev. Lett. 85 (2000) 3100'

[20] K. Adcox et al. (PHENIX Collaboration), Phys. Rev. Lett. 86 (2001) 3500 
[21] C. Adler et al. (STAR Collaboration), 'P

[22] B. B. Back et al. (PHOBOS Collaboration), nunci-ex/0108009!

[23] B. B. Back et al. (PHOBOS Collaboration), 'Phys. Rev. Lett. 87. (2001) 102303"

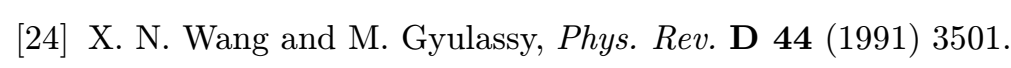

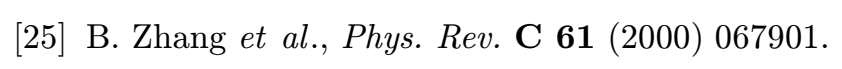

[26] J. D. Bjorken, Pיhys. Rev.

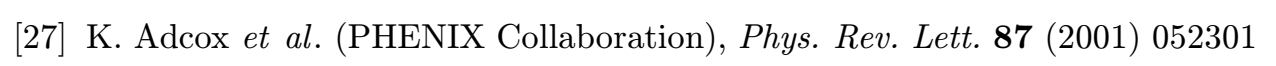

[28] T. Alber et al. (NA49 Collaboration), Phhys. Rev. Lett. 75 (1995) 3814.

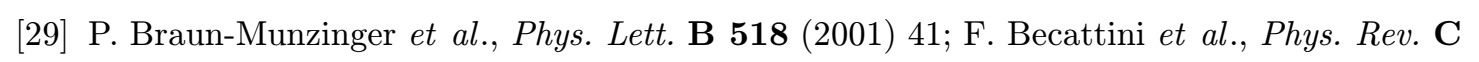
-

[30] J. Sollfrank and U. Heinz, Quark Gluon Plasma 2, ed. R. C. Hwa, World Scientific, 1996, p. 555.

[31] P. Braun-Munzinger, nucuci-ex/0007021i:

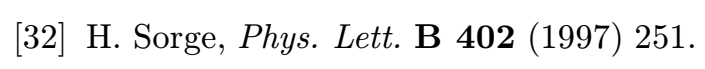

[33] A. M. Poskanzer and S. A. Voloshin, P'hys. Rev. $\mathbf{C} \overline{8}(19 \overline{9} \overline{8})$ 1671;

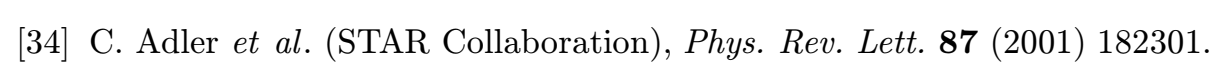

[35] P. Huovinen et al., Physs. Lett. B

[36] M. Gyulassy and M. Plümer, 'Phys. Lett. B 2043 (1990) -

[37] X. N. Wang and M. Gyulassy, iPhys. Rev. Lett. 68 (1992) $14 \overline{4} \overline{0} ;$ X. N. Wang, 'Phys. Rev. - $58(1998) 2321$.

[38] R. Baier, D. Schiff and B. G. Zakharov, Ann. Rev. Nucl. Part. Sci. 50 $\mathbf{0}(2000)$

[39] C. Albajar et al. (UA1 Collaboration), 'Nucl. Phys. B-335_(1990) $26 \overline{1}$.

[40] D. Antreasyan et al., Phys. Rev. D.19-1979) 764 .

[41] A. Drees, Proceedings of Quark Matter 2001, Stony Brook, N.Y., Jan. 15-21, 2001. Nucl. Phys. A, in press.; 'nucl-ex/0105019'

[42] K. Adcox et al. (PHENIX Collaboration), nnucl-ex/109003.

[43] I. Vitev and M. Gyulassy, nuuci-th/0104066.

[44] P. Huovinen et al., Phys. Lett.

[45] M. Gyulassy, I. Vitev and X. N. Wang, Phys. Rev. Lett. 86 $(2001) 2537$. 